\title{
The Cygnus region of the galaxy: A VERITAS perspective
}

\author{
A. Weinstein for the VERITAS Collaboration ${ }^{\mathrm{a}}$ \\ Department of Physics and Astronomy, Iowa State University, Ames, IA 50011, USA
}

\begin{abstract}
The Cygnus-X star-forming region ("Cygnus") is the richest star-forming region within $2 \mathrm{kpc}$ of Earth and is home to a wealth of potential cosmic ray accelerators, including supernova remnants, massive star clusters, and pulsar wind nebulae. Over the past five years, discoveries by several gamma-ray observatories sensitive in different energy bands, including the identification by Fermi-LAT of a potential cocoon of freshly accelerated cosmic rays, have pinpointed this region as a unique laboratory for studying the early phases of the cosmic ray life cycle. From 2007 to 2009 VERITAS, a very high energy (VHE; E > $100 \mathrm{GeV}$ ) observatory in southern Arizona, undertook an extensive survey of the Cygnus region from 67 to 82 degrees Galactic longitude and from -1 to 4 degrees in Galactic latitude. In the years since, VERITAS has continued to accumulate data at specific locations within the survey region. We will review the discoveries and insights that this rich dataset has already provided. We will also consider the key role that we expect these data to play in interpreting the complex multiwavelength picture we have of the Cygnus region, particularly in the vicinity of the Cygnus cocoon. As part of this discussion we will summarize ongoing studies of VERITAS data in the Cygnus region, including the development of new data analysis techniques that dramatically increase VERITAS' sensitivity to sources on scales larger than a square degree.
\end{abstract}

\section{Introduction}

Supernova remnants (SNRs) are believed to produce the bulk of Galactic cosmic rays up to the "knee" at $10^{15} \mathrm{eV}$, a theory supported by recent $\mathrm{GeV}$ and $\mathrm{TeV} \gamma$-ray and $\mathrm{X}$-ray observations [1-5]. However, so far conclusive evidence for protons being accelerated all the way to the knee by SNR shocks has been elusive. Recent theoretical models indicate that SNRs are PeVatrons for only a brief portion of their life cycle (as little as 30 years) and that the highest energy protons escape early [6]. The number of nearby historical supernova remnants is small and the number of potential PeVatrons even smaller. It is therefore interesting to hunt for other sources of gamma-ray emission that can be identified with populations recently-accelerated cosmic rays, whether these are cosmic rays recently escaped from their accelerator or the result of the collective action of multiple hadronic cosmic ray accelerators (e.g. superbubbles).

From this point of view, the Cygnus region-rich both in potential VHE gamma-ray sources and likely cosmic ray accelerators, including a superbubble-was a natural choice for a VHE gamma-ray survey by the VERITAS observatory. Cygnus is known to be the richest starforming region within $2 \mathrm{kpc}$ of Earth and to contain high mass of molecular gas (more than ten times that of its neighboring star-forming regions combined) [7]. It contains a treasure trove of massive stars in stellar nurseries, young open clusters, and OB associations, two of which-Cyg OB1 and OB2 [8]-are of particular interest. The EGRET catalog as well as known supernova

a e-mail: amandajw@iastate.edu remnants, pulsar wind nebulae, and high-mass $\mathrm{x}$-ray binaries also highlighted potential gamma-ray source candidates.

\section{Survey strategy and initial results}

In April 2007 VERITAS began a two-year survey of the portion of the Cygnus region of the Galactic Plane between $67^{\circ}<l<82^{\circ}$ and $-1^{\circ}<b<4^{\circ}$. The survey consisted primarily of overlapping 20-minute observations on a grid of points with $0.8^{\circ}$ separation in Galactic latitude and $1^{\circ}$ separation in galactic longitude, coupled with targeted follow-up observations and serendipitous observations of transients. This strategy provided a fairly uniform effective exposure of $\sim 6$ hours across the entire survey area, plus smaller regions with significantly enhanced exposure [9].

Details of the preliminary survey analysis, based on all VERITAS data in the region through Fall 2009, are given in $[9,10]$. Figure 1 shows the resulting VERITAS background-subtracted sky map of the portion of the survey region with $74^{\circ} \leq l<82^{\circ}$. Clear detections were obtained of two moderately extended sources: the wellestablished VHE gamma-ray source TeV J2032+4130 and a newly-discovered patch of VHE gamma-ray emission, VER J2019+407, overlapping the northwest rim of SNR G78.2+2.1. No other significant source detections were found in the survey region.

\subsection{Multiwavelength context}

The multiwavelength picture of the region, particularly in gamma rays, has evolved significantly since the inception

This is an Open Access article distributed under the terms of the Creative Commons Attribution License 4.0, which permits unrestricted use, distribution, and reproduction in any medium, provided the original work is properly cited. 


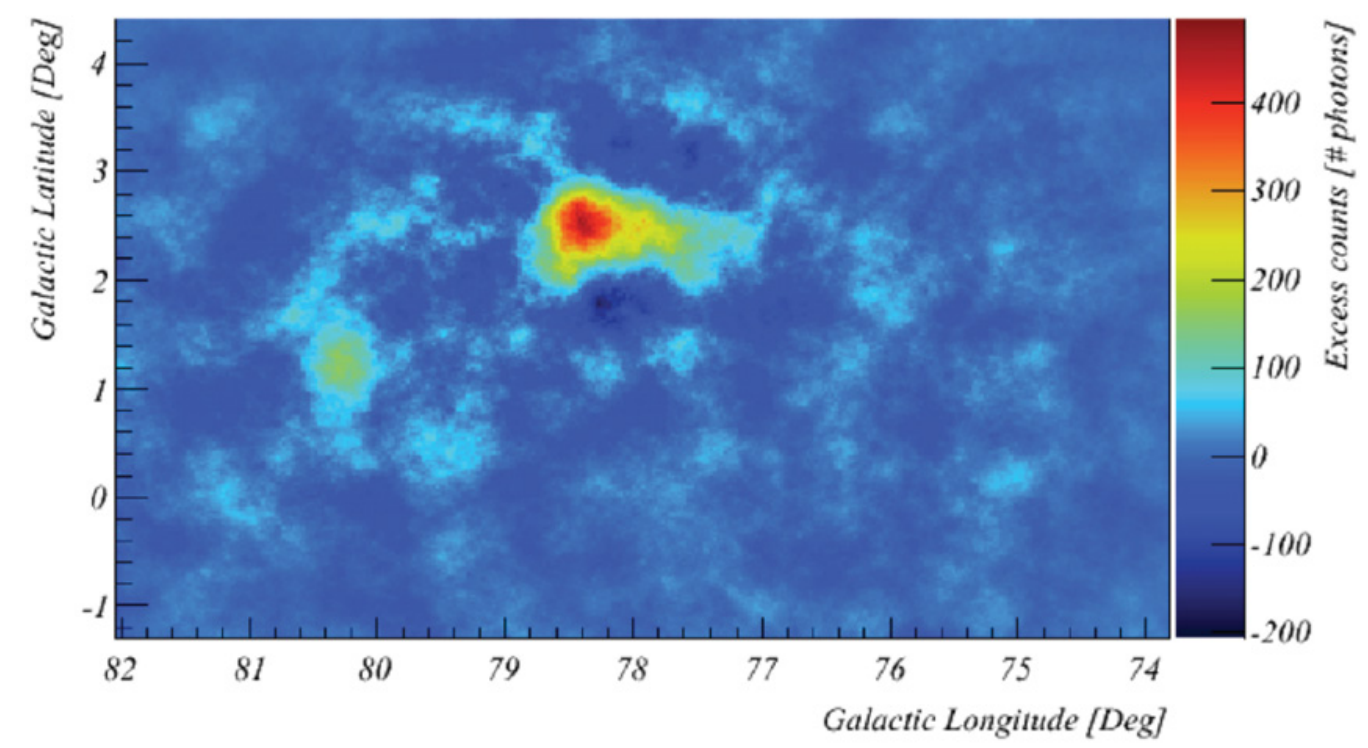

Figure 1. A background-subtracted photon excess map of the portion of the VERITAS Cygnus region survey with $1>74^{\circ}$, using all data taken through November 2009. This is a slightly modified version of the map shown in [9].

of the VERITAS survey. This is particularly true for the two regions associated with Cyg OB2 and Cyg OB1. Each of these regions contains highly extended gammaray sources detected at median energies of $\sim 12 \mathrm{TeV}$ by the Milagro Gamma Ray Observatory as well as gammaray pulsars detected by the Fermi Gamma-ray Space Telescope (Fermi LAT). Most significantly, the region around Cyg OB2 also contains a large region of hardspectrum gamma-ray emission observed by Fermi LAT above $3 \mathrm{GeV}$ (1FHL J2028.6+4110e) [11,12]. Because the emission fills a cavity carved in the ISM by stellar winds from a nearby collection of $\mathrm{OB}$ stars, it is frequently dubbed the "Cygnus cocoon" for convenience. We can infer from the gamma-ray spectrum between $3 \mathrm{GeV}$ and $500 \mathrm{GeV}$ that the generating cosmic ray spectrum is quite hard, which suggests the cosmic rays within the cocoon are freshly accelerated [11,12]. Ackermann et al. [11] argue strongly that the Cygnus cocoon is evidence for cosmic-ray acceleration due to collective action of massive stellar winds within a superbubble. However, contributions from SNR G78.2+2.1 and the Cyg OB2 association, while disfavored, are not completely ruled out.

Measurements of the cocoon spectrum and energydependent morphology at energies above a few hundred $\mathrm{GeV}$ are a critical input when differentiating between scenarios, both in terms of the type of accelerated particles and their source. Figures 2 and 3 illustrate the most current knowledge of the cocoon in gamma rays between a $\mathrm{GeV}$ and $10 \mathrm{TeV}$ [13]. The cocoon is co-located with an extended source of $>10 \mathrm{TeV}$ gamma rays seen by Milagro (MGRO J2031+41) [14,17]. A recently-updated measurement by ARGO-YBJ shows a similarly extended gamma ray source at intermediate energies. Figure 3 shows the spectrum of the cocoon as measured by all three experiments $[11-14,17]$. The spectrum is well-fit by a pure hadronic emission model with a primary proton energy cutoff somewhere between 40 and $150 \mathrm{TeV}$ and

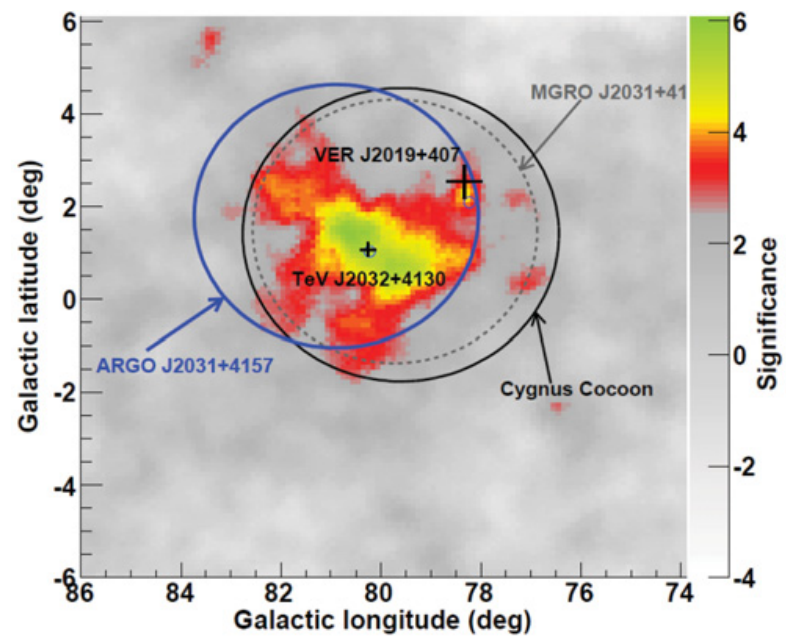

Figure 2. ARGO-YBJ significance map around the Cygnus Cocoon. Large circles denote the positions and $68 \%$ containment regions of ARGO J2031+4157 (blue) [13], MGRO J2031+41 (black) [14] and the Cygnus cocoon (dashed black) [11]. Crosses denote the position and extension of TeV J2032+4130 [15], VER J2019+407 [16]; small circles denote the positions of pulsars PSR J2021+4026 and PSR J2032+4127. Reproduced from [13].

remains consistent with the superbuble scenario advanced in [11]. However, this spectrum is still subject to significant statistical and systematic uncertainties. In particular, ARGO-YBJ's angular resolution means that they can only directly measure the combined emission produced by VER J2019+407, TeV J2032+4130, and the Cygnus cocoon. They must rely on measurements from VERITAS and other IACTs to subtract off the contributions from VER J2019+407 and TeV J2032+4130 [13]. VERITAS in principle has the angular resolution to separately measure these contributions, but, as previously noted, does not at this time detect the cocoon emission. 


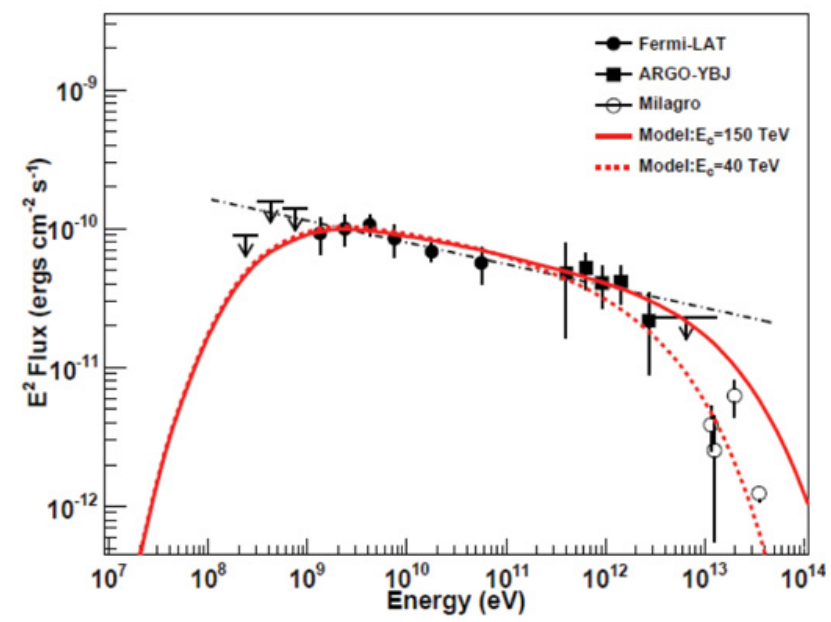

Figure 3. Current constraints on the cocoon spectrum from Fermi LAT (filled points) [11], ARGO-YBJ (filled squares), and Milagro (hollow circles). Arrows denote Fermi LAT upper limits; the MGRO J2031+41 flux points are at 12, 20, and $35 \mathrm{TeV}$ $[14,17,18]$. The fourth hollow circle, below the first at $12 \mathrm{TeV}$, has had the TeV J2032+4130 flux subtracted. The black dotdashed line indicates a power-law fit to the combined Fermi LAT and ARGO-YBJ spectrum; the red lines are hadronic model fits to the combined data assuming proton cutoff energies at $150 \mathrm{TeV}$ (solid) and $40 \mathrm{TeV}$ (dashed). Reproduced from [13].

\section{VERITAS near MGRO J2031+41}

\subsection{VER J2019+407}

As noted earlier, the VERITAS Cygnus region survey led to the discovery of VER J2019+407, a compact $(\sigma \approx$ $0.23^{\circ}$ ) region of gamma-ray emission above a few hundred GeV. VER J2019+407 overlaps the brightest part of the northern radio shell of SNR G78.2+2.1, also known as the $\gamma$ Cygni SNR [16]. This radio, X-ray, and gammaray SNR [7,19-21] is believed to be in an early phase of adiabatic expansion into a low-density medium. The radio and X-ray emission reveal a shell-like structure $\sim 1^{\circ}$ across with high-intensity features to the north and south $[22,23]$. By contrast, the hard (power-law spectral index $2.39 \pm 0.14$ ) gamma-ray emission detected by Fermi LAT (1FHL J2021.0+4031e) between $10 \mathrm{GeV}$ and $500 \mathrm{GeV}$ is well-represented by a featureless disk slightly larger than the radio remnant $[11,12,21]$. The remnant is thought to be at a distance of $\sim 1.7 \mathrm{kpc}$. The center of the $\gamma$ Cygni SNR boasts a low-luminosity gamma-ray pulsar, PSR J2021+4026, which may or may not be the remnant of the $\gamma$ Cygni SNR's progenitor star [25-27].

It is plausible that VER J2019+407 originates from protons and heavier nuclei accelerated in the SNR shock, as argued in [16]. Yet this interpretation is by no means iron-clad and a number of puzzles remain to be addressed. VER J2019+407 is peculiarly compact, given that highintensity radio features are visible in both the north and south and the only indications of molecular material lie in the portion of the shell opposite to VER J2019+407. The discrepancy in size between 1FHL J2021.0+4031e and VER J2019+407 is even more striking, given that a naive power-law extrapolation of 1FHL J2021.0+4031e up to

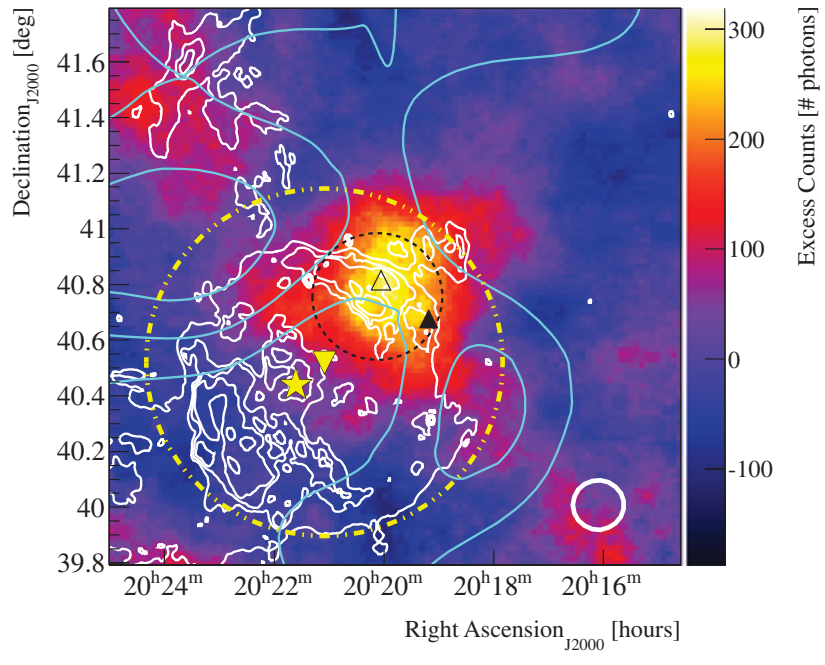

Figure 4. Background-subtracted gamma-ray counts map showing VER J2019+407 and its fitted extent (black dashed circle). The radio remnant is traced by Canadian Galactic Plane Survey (CGPS) $1420 \mathrm{MHz}$ continuum contours at brightness temperatures of $23.6 \mathrm{~K}, 33.0 \mathrm{~K}, 39.6 \mathrm{~K}, 50 \mathrm{~K}$ and $100 \mathrm{~K}$ (white) [24]. The star symbol shows the location of PSR J2021+4026. The fitted centroid and extent 1FHL J2021.0+4031e are indicated by the inverted triangle and dot-dashed circle (yellow). The open and filled triangles (black) show the positions of the Fermi LAT catalog sources 1FGL J2020.0+4049 and 2FGL J2019.1+4040, now subsumed into the extended $\mathrm{GeV}$ emission from the entire remnant [21]. The $0.16,0.24$, and 0.32 photons bin ${ }^{-1}$ contours of the Fermi LAT detection of the Cygnus cocoon are shown in cyan [11]. The VERITAS gamma-ray PSF is shown for comparison (white circle, bottom right). Reproduced from [16].

$1 \mathrm{TeV}$ should suggests a much larger portion of the SNR should be detectable by VERITAS.

This puzzle admits at least two competing physical solutions. First, the spectra of different regions of the $\gamma$ Cygni SNR may evolve differently above $500 \mathrm{GeV}$, with the emission from the northern shell having a higher-energy cutoff than the remainder of the remnant. This portion of the remnant would consequently appear brighter at higher energies. The effect could be accentuated by systematic effects in the data analysis arising from the size of the $\gamma$ Cygni SNR and the presence of a magnitude- 2 star overlapping the southeastern portion of the shell. Such effects would dilute VERITAS' sensitivity to emission from the entire SNR. This scenario is nicely consistent with the fact both 1FHL J2021.0+4031e and VER J2019+407 have measured spectra consistent with a power-law spectrum with $\Gamma=2.4$. A lessfavored interpretation is that VER J2019+407 is a chance superposition of a PWN in the $\gamma$ Cygni SNR line of sight, possibly associated with a recently-discovered X-ray point source nearby [28].

\section{2. $\mathrm{TeV} \mathrm{J} 2032+4130$}

The HEGRA collaboration first discovered the VHE gamma-ray source TeV J2032+4130 in 2002 [29,30]. The detection and source extension were later confirmed by IACT observatories MAGIC and VERITAS, which also 
provided more precise measurements of its spectrum. All instruments find a power-law spectrum consistent with $\Gamma=2.1$, although VERITAS and MAGIC disagree slightly as to the flux normalization [15,31]. No evidence of either an energy-dependent morphology or a spectral cutoff (up to $20 \mathrm{TeV}$ ) has been seen thus far [15,31]. The source overlaps a gamma-ray pulsar, PSR J2032+4127. When Cordes and Lazio [32] models for dispersion in the Milky Way are applied to recent radio observations [33] of PSR J2032+4127, they place it at $3.6 \mathrm{kpc}$, beyond Cyg OB2. Other models place it at $1.7 \mathrm{kpc}$, consistent with standard distance estimates for both Cyg OB2 and the $\gamma$ Cygni SNR [33].

Subsequent to the survey, VERITAS undertook a set of deep observations of $\mathrm{TeV} \mathrm{J} 2032+4130$, reported on in Aliu et al. [31]. The gamma-ray emission appears to be confined to one of the rare voids in bright diffuse radio and infrared emission seen from Cygnus. Aliu et al. [31] argue that the void could be due to a long-ago ( $>30 \mathrm{kyr}$ ) supernova explosion, with TeV J2032+4130 being a relic PWN powered by PSR J2032+4127 [25]. It remains to be seen what implications the recent timing studies of PSR $\mathrm{J} 2032+4127$, which point to its being part of a binary system, have for this theory [34]. An alternate scenario that has $\mathrm{TeV} \mathrm{J} 2032+4130$ powered by winds from OB stars in the Cyg OB2 association cannot be ruled out, but is less attractive given the paucity of massive OB stars overlapping the observed VHE gamma-ray emission.

\subsection{The Cygnus cocoon}

At first glance VERITAS' failure to detect the Cygnus cocoon, clearly seen by ARGO-YBJ, is puzzling. A straightforward folding of the measured cocoon spectrum with the VERITAS response functions suggests that VERITAS should detect a substantial number of photons from the Cygnus cocoon above a few hundred $\mathrm{GeV}$, more than enough for a clear detection. However, the two results are consistent when the limitations of the standard VERITAS data analysis methods are taken into account. The reflected region model and ring background model [35] both estimate the cosmic-ray background level using regions of the field of view away from the $\gamma$-ray source. When a source fills a large fraction of the VERITAS field of view, it becomes impossible to both exclude the source and select an adequate background estimation region that is not photon-contaminated. When the source is not excluded portions of the source itself will be incorporated into the background estimation. If the source is sufficiently large it will self-subtract and become undetectable. Figure 5 illustrates this effect for a toy model simulation of VERITAS observations of the cocoon region. For the sake of simplicity, this study was limited to photon candidate energies between $500 \mathrm{GeV}$ and $1 \mathrm{TeV}$. The four primary sources in the region-the $\gamma$ Cygni SNR, TeV J2032+4130, VER J2019+407, and the Cygnus cocoonare modeled as Gaussians with power-law spectra, with the relevant parameters either taken directly or extrapolated from previous measurements $[11,12,16,21,31]$. Although the modeled exposure is increased with respect to both the 2009 survey analysis and the current VERITAS archival data set, the standard ring background model analysis still

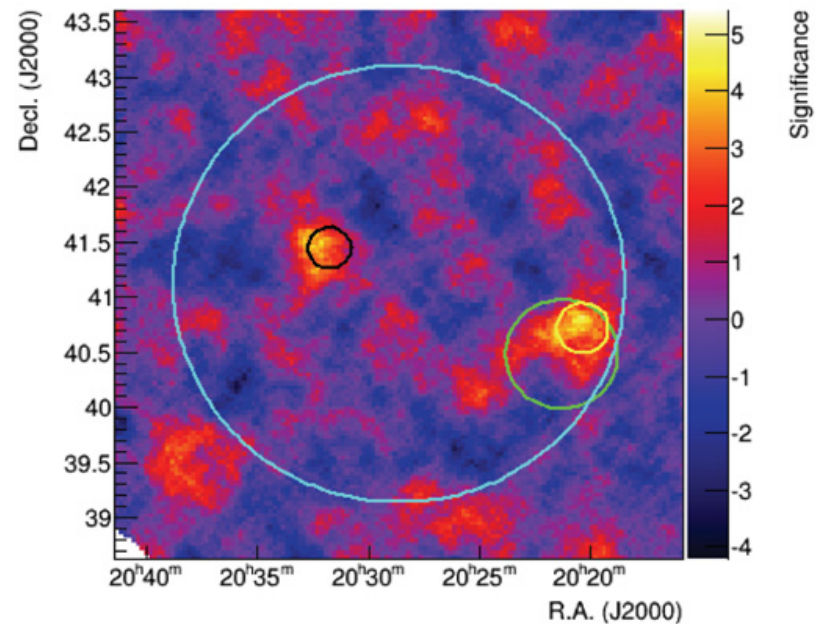

Figure 5. Significance map produced by applying ring background model analysis to a toy model simulation of $\sim 200$ hours of VERITAS observations of the Cygnus cocoon region. (Caveat: while this illustrates the relevant principle it is not a precise analog of the VERITAS survey exposure as of 2009).

reveals notable $(>4 \sigma)$ excesses only for VER J2019+407 and TeV J2032+4130.

\subsection{Future prospects}

As noted in the preceding sections, VERITAS has continued to accumulate data in the Cygnus region. Two different studies using all VERITAS data to date in the Cygnus region are planned. One is an updated version of the standard analysis used to obtain the preliminary survey results. The other approach uses a maximum likelihood method designed to enhance VERITAS' sensitivity to highly extended sources such as the Cygnus cocoon. This "3D" maximum likelihood method (3D MLM) describes the data in terms of two spatial position coordinates, a parameter known as mean-scaled width (MSW) that is used to distinguish between gamma rays and cosmic rays, and energy. The fit is extended and unbinned in three of the four variables, leading us to refer to the maximum likelihood as "three-dimensional" or "3D." The inclusion of MSW as a parameter in the fit permits extended gammaray sources that fill the field of view to be distinguished from the cosmic ray background, even when the two have strikingly similar spatial distributions. The effectiveness of the technique is easily demonstrated by applying it to the toy model simulation discussed in Sect. 3.3. The 3D MLM detects significant emission $(\sqrt{T S}>30)$ from the simulated cocoon and the simulated $\gamma$-Cygni SNR. Figure 6 shows that even with minimal smoothing, a broad region of $\gamma$-ray excess corresponding to the cocoon appears in the spatial residual map.

\section{Disentangling MGRO J2019+307}

MGRO J2019+37, in the vicinity of Cyg OB1, is the brightest Milagro source in the Cygnus region, with a flux of about $80 \%$ of the Crab Nebula flux at $20 \mathrm{TeV}$ and a bright core over $1^{\circ}$ in extent [14]. A campaign of deep 


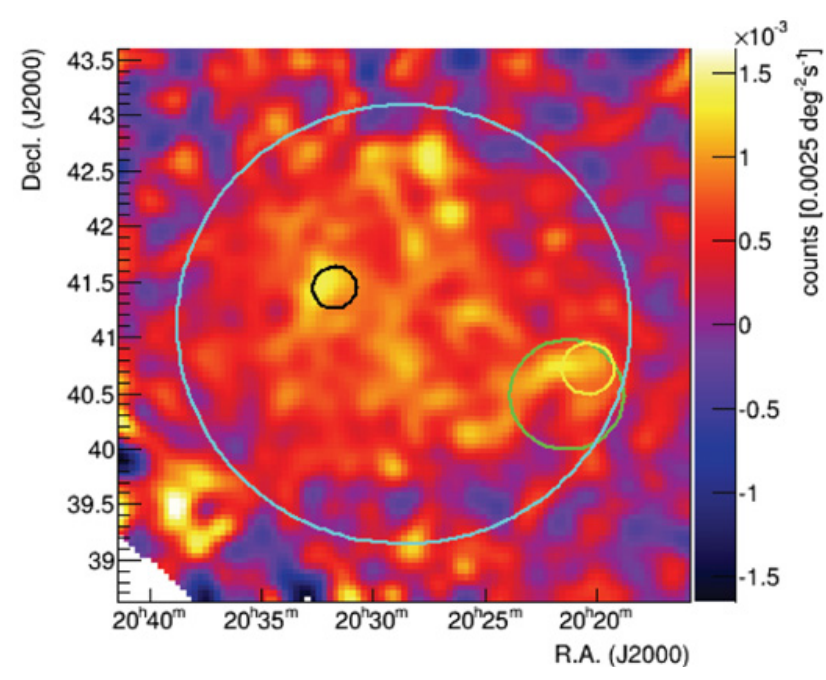

Figure 6. Exposure-corrected 3D MLM spatial residual map for the same simulated dataset, projected in the signal dominated region $0.05<M S W<1.1$. The map has been smoothed by the VERITAS PSF. In both figures 1- $\sigma$ contours are shown for the Gaussian source models: the cocoon (dashed blue), $\gamma$ Cygni SNR (short-dashed green), VER J2019+407 (yellow), and TeV J2032+4130 (black).

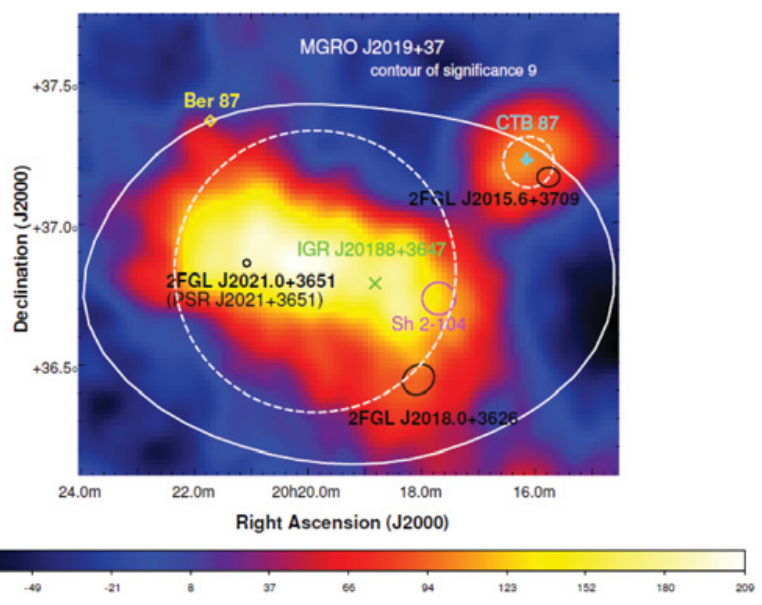

Figure 7. Map of the gamma-ray excess above $600 \mathrm{GeV}$, seen by VERITAS in the vicinity of MGRO J2019+37. The color bar indicates the number of excess events within a $0.23^{\circ}$ search radius. White dashed circles indicate the regions used to extract the spectra of VER J2016+371 and VER J2019+368. The $9 \sigma$ significance contour of MGRO J2019+37 is overlaid in solid white. The remaining solid ellipses, diamonds and crosses indicate the locations of potential counterparts. Reproduced from [36].

observations targeting MGRO J2019+37 revealed gammaray emission not detected in the original survey and strongly suggest that MGRO J2019+37, rather than being a unique source, is a synthesis of multiple contributions. Figure 7 shows VERITAS' current best picture of MGRO $\mathrm{J} 2019+37$ and its vicinity between $600 \mathrm{GeV}$ and $10 \mathrm{TeV}$ [36].

Thus far VERITAS has identified two sources in this region. One, VER $\mathrm{J} 2016+371$, is point-like and

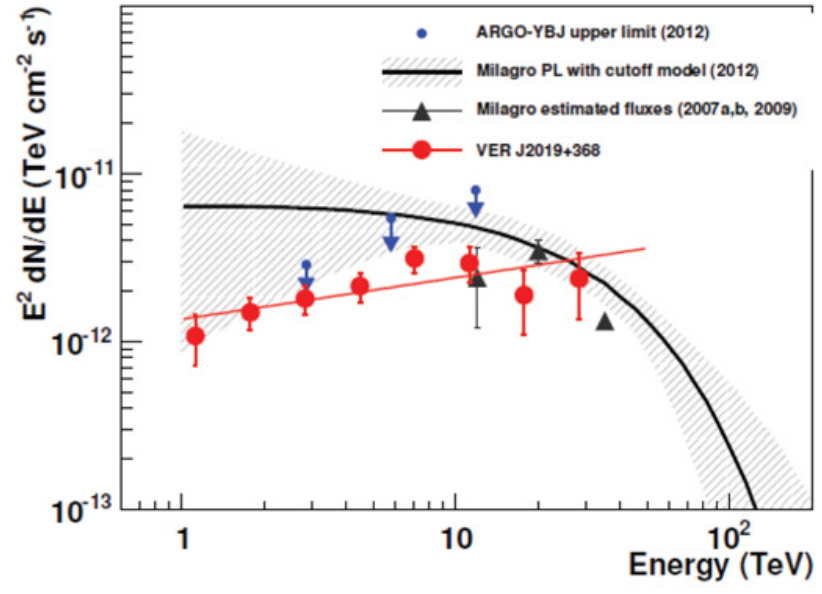

Figure 8. Spectral energy distribution of MGRO J2019+37 and VER J2019+368. The VER J2019+368 spectrum from $1 \mathrm{TeV}$ to almost $30 \mathrm{TeV}$ (red dots) is best fit with a power law with $\Gamma=1.75 \pm 0.3$. The original Milagro flux points are shown at 12, 20 and $35 \mathrm{TeV}$ (black triangles) [17,37,38]. The black curve shows the 2012 best-fit spectrum for MGRO J2019+37: a power law with a cutoff [14]. The shadowed area corresponds to the $1 \sigma$ band. ARGO-YBJ 90\% confidence-level upper limits for MGRO J2019+37 are shown with blue arrows [39]. Reproduced from [36].

corresponds best with SNR CTB 87. CTB 87's radio morphology buttresses VER J2016+371's identification as a relic PWN, as does the presence of pulsar candidate CXOU J201609.2+371110 within the radio contours [36]. The other, VER $\mathrm{J} 2019+368$, is $\sim 1^{\circ}$ ridge of diffuse emission, roughly bounded by the bright bubble $\mathrm{H}$ II region Sh 2-104 to the west and the energetic gamma-ray and radio pulsar PSR J2021+3651 to the east. Figure 8 shows that the VER J2019+368 spectrum plausibly dominates that of MGRO J2019+37 at high energies, particularly when the ARGO-YBJ upper limits are taken into account. However, VER J2019+368 itself likely incorporates emission from several unresolved sources. These may include the PWN of PSR J2021+3651 and the H II region Sh 2-104.

\section{Future outlook}

The recent surveys of the Cygnus region by VERITAS, Milagro, ARGO-YBJ and Fermi LAT have filled in a complex and fascinating picture in gamma rays from $1 \mathrm{GeV}$ to several tens of $\mathrm{TeV}$. Vivid as this picture is, parts of it remain fuzzy and incomplete. In the short term, we hope to make further advances by combining current and future VERITAS observations of this region with new data analysis techniques and by leveraging the synergy between VERITAS, Fermi LAT and Milagro's more sensitive successor, the recently-commissioned water Cherenkov observatory HAWC. In the longer term, the northern half of the next-generation IACT observatory the Cherenkov Telescope Array (CTA) is perfectly suited to studies of this challenging portion of the gamma-ray sky. 
The VERITAS 3D MLM development has been supported in part by NASA grant NNX11A086G. VERITAS is supported by grants from the U.S. Department of Energy Office of Science, the U.S. National Science Foundation and the Smithsonian Institution, by NSERC in Canada, and by Science Foundation Ireland (SFI 10/RFP/AST2748). We acknowledge the excellent work of the technical support staff at the Fred Lawrence Whipple Observatory and at the collaborating institutions in the construction and operation of the instrument.

\section{References}

[1] F. Giordano, M. Naumann-Godo, J. Ballet, et al., ApJ 744, L2 (2012), 1108.0265

[2] V.A. Acciari, E. Aliu, T. Arlen, et al., ApJ 730, L20, (2011), 1102.3871

[3] K.A. Eriksen, J.P. Hughes, C. Badenes, et al., ApJ 728, L28, (2011), 1101.1454

[4] A. Atoyan and C.D. Dermer, ApJ 749, L26, (2012)

[5] M. Ackermann, M. Ajello, A. Allafort, et al., Science 339, 807 (2013) 1302.3307

[6] I. Reichardt, E. de Oña-Wilhelmi, J. Rico, et al., A\&A 546, A21, (2012), 1207.2057

[7] J.-M. Le Duigou and J. Knödlseder, A\&A 392, 869, (2002)

[8] A. Konopelko, R.W. Atkins, G. Blaylock, et al., ApJ 658, 1062 (2007), astro-ph/0611730

[9] A. Weinstein, for the VERITAS Collaboration, arXiv e-prints (2009), 0912.4492

[10] A. Weinstein, f.t.V. Collaboration, arXiv.org astroph. HE (2009)

[11] M. Ackermann, M Ajello, A Allafort, et al., Science 334, 1103 (2011)

[12] The Fermi-LAT Collaboration, ArXiv e-prints (2013), 1306.6772

[13] B. Bartoli, P. Bernardini, X.J. Bi, et al., ApJ 790, 152, (2014)

[14] A.A. Abdo, U. Abeysekara, B.T. Allen, et al., ApJ 753, 159 (2012), 1202.0846

[15] J. Albert, E. Aliu, H. Anderhub, et al., ApJ 675, L25 (2008), 0801.2391

[16] E. Aliu, S. Archambault, T. Arlen, et al., ApJ 770, 93, (2013), 1305.6508

[17] A.A. Abdo, B.T. Allen, T. Aune, et al., ApJ 700, L127, (2009), 0904.1018
[18] A.A. Abdo, B Allen, T Aune, et al., ApJ 688, 1078 (2008)

[19] L.A. Higgs, T.L. Landecker, and R.S. Roger, AJ 82, 718 (1977)

[20] T.A. Lozinskaya, V.V. Pravdikova, and A.V. Finoguenov, Astronomy Letters 26, 77 (2000)

[21] J. Lande, M. Ackermann, A. Allafort, et al., ApJ 756, 5 (2012), 1207.0027

[22] X. Zhang, Y. Zheng, T.L. Landecker, and L.A. Higgs, A\&A 324, 641 (1997)

[23] Y. Uchiyama, T. Takahashi, F.A. Aharonian, and J.R. Mattox, ApJ 571, 866 (2002), astro-ph/0202414

[24] A.R. Taylor, S.J. Gibson, M. Peracaula, et al., AJ 125, 3145 (2003)

[25] A.A. Abdo, M. Ackermann, M. Ajello, et al., ApJS 187, 460 (2010), 0910.1608

[26] P.L. Nolan, A.A. Abdo, M. Ackermann, et al., ApJS 199, 31 (2012), 1108.1435

[27] L. Trepl, C.Y. Hui, K.S. Cheng, et al., MNRAS 405, 1339 (2010), 1008.4313

[28] D.A. Leahy, K. Green, and S. Ranasinghe, MNRAS 436, 968 (2013), 1307.3151

[29] F. Aharonian, A. Akhperjanian, M. Beilicke, et al., A\&A 393, L37 (2002), astro-ph/0207525

[30] F. Aharonian, A. Akhperjanian, M. Beilicke, et al., A\&A 431, 197 (2005)

[31] E. Aliu, T. Aune, B. Behera, et al., ArXiv e-prints (2014), 1401.2828

[32] J.M. Cordes and T.J.W. Lazio, ArXiv Astrophysics e-prints (2002), astro-ph/0207156

[33] F. Camilo, P.S. Ray, S. M. Ransom, et al., ApJ 705, 1 (2009), 0908. 2626

[34] A. Lyne, B. Stappers, M. Keith, et al., ArXiv e-prints (2015), 1502.01465

[35] D. Berge, S. Funk, and J. Hinton, A\&A 466, 1219 (2007) [arXiv: astro-ph/0610959]

[36] E. Aliu, T. Aune, B. Behera, et al., ApJ 788, 78 (2014)

[37] A.A. Abdo, B. Allen, D. Berley, et al., ApJ 658, L33 (2007) [astro-ph/0611691]

[38] A.A. Abdo, B. Allen, D. Berley, et al., ApJ 664, L91 (2007), 0705.0707

[39] B. Bartoli, P. Bernardini, X.J. Bi, et al., ApJ 745, L22, (2012), 1201.1973 